\title{
Estudos de Fidedignidade para o Desenho da Família Cinética
}

\author{
Carolina Ferreira Barros Klumpp ${ }^{1}$ \\ Centro Universitário FIEO, Osasco-SP, Brasil \\ Manuela Vilar, Marcelino Pereira \\ Universidade de Coimbra, Coimbra, Portugal \\ Márcia Siqueira de Andrade \\ Centro Universitário FIEO, Osasco-SP, Brasil
}

\section{RESUMO}

Esta pesquisa teve como objetivo realizar estudos de fidedignidade para o Desenho da Família Cinética, sendo estes relativos aos parâmetros de fidedignidade entre avaliadores, intra-avaliador e estabilidade temporal/teste-reteste. Os instrumentos utilizados foram o Desenho da Família Cinética e o Teste das Matrizes Progressivas Coloridas de Raven. Participaram desses estudos dois juízes e 66 crianças, sendo 16 com problemas de aprendizagem. Para a análise de dados, utilizou-se o coeficiente kappa de Cohen, a correlação $\rho$ de Spearman e a diferença estatística entre as médias dos pontos por meio do Teste Wilcoxon. Os resultados mostraram índices substanciais a perfeitos de concordância nos estudos de fidedignidade entre avaliadores e intra-avaliador para todos os itens de análise do Desenho da Família Cinética e, no teste-reteste, não houve diferenças estatisticamente significativas entre as médias das pontuações entre as duas aplicações. Conclui-se pela robustez psicométrica do Desenho da Família Cinética, em termos de indicadores de fidedignidade.

Palavras-chave: desenho da família cinética; técnicas autoexpressivas; fidedignidade.

\section{ABSTRACT - Reliability studies for the Kinetic Family Drawing}

This study aimed to perform reliability analyses for the Kinetic Family Drawing, in relation to inter- and intra-rater reliability parameters and temporal/test-retest stability. The instruments used were the Kinetic Family Drawing and the Raven Colored Progressive Matrix Test. Twelve judges and 66 children participated in this study, 16 of whom had learning problems. For the data analysis, Cohen's kappa coefficient, Spearman's correlation coefficient $\rho$, and the statistical difference between the means of the points through the Wilcoxon test were used. The results showed substantial perfect agreement indices in the inter- and intra-rater reliability analyses for all items of the Kinetic Family Drawing, and in the test-retest there were no statistically significant differences between the mean scores of the two applications. The psychometric robustness of the Kinetic Family Drawing, in terms of reliability indicators, was confirmed. Keywords: Kinetic family drawing; Self-expressive techniques; Reliability.

\section{RESUMEN - Estudios de confiabilidad para el Dibujo Kinético de la Familia}

Esta investigación tuvo como objetivo realizar estudios de confiabilidad para el Dibujo Kinético de la Familia, que se relaciona con los parámetros de referencia entre los evaluadores, intra-evaluadores y estabilidad temporal/test-retest. Los instrumentos utilizados fueron el Dibujo Kinético de la Familia y la Test de Matrices Progresivas de Raven. Participaron de los estudios 2 jueces y 66 niños, siendo que 16 de ellos poseían problemas de aprendizaje. Para el análisis de los datos, se utilizó el coeficiente kappa de Cohen, la correlación de Spearman $\rho$ y la diferencia estadística entre las medias de los puntos por medio de la prueba de Wilcoxon. Los resultados mostraron índices de concordancia considerables en los estudios de confiabilidad entre evaluadores e intra-evaluadores para todos los ítems de análisis del Dibujo Kinético de la Familia y, en el test-retest, no hubo diferencias estadísticamente significativas entre las medias de las puntuaciones entre las dos aplicaciones. Se concluye por la robustez psicométrica del Dibujo Kinético de la Familia, en términos de indicadores de confiabilidad.

Palabras clave: Dibujo Kinético de la Familia; Técnicas autoexpresivas; Confiabilidad.

O Desenho da Família Cinética (Kinetic Family Drawing - KFD; Burns \& Kaufman, 1972) é uma técnica autoexpressiva que se utiliza do grafismo para avaliar as percepções da criança sobre as relações interpessoais de sua família (Tharinger \& Stark, 1990). A consigna dessa técnica consiste no ato da criança desenhar sua família fazendo algo. O comando do movimento (a dimensão "cinética") no desenho permite à criança revelar o que é

${ }^{1}$ Endereço para correspondência: Departamento de Pós-Graduação em Psicologia Educacional do Centro Universitário FIEO. Avenida Franz Voegeli, 300, Vila Yara, 06020-190, Osasco, SP. Tel.: (11) 3651-9999. E-mail: carolfbk@gmail.com

Agradecimento ao apoio da CAPES (Coordenação de Aperfeiçoamento de Pessoa de Nível Superior). 
mais representativo sobre a dinâmica e o vínculo familiar em sua produção, bem como torna a consigna mais complexa, uma vez que requer por parte da criança um maior planejamento, decidindo quem incluir no desenho, fazendo o quê e com quem. O dinamismo passa a ser, desse modo, a base da representação, pois ao invés de se observar apenas o objeto e o movimento orientado desse objeto, a observação evolui para o movimento e o contato com o objeto (Mercado, (s.f); Tharinger \& Stark, 1990).

$\mathrm{O}$ KFD, assim como as demais técnicas projetivas gráficas, é visto como não ameaçador, pois a atividade de desenhar diminui a ansiedade da criança e a coloca à vontade para se expressar. Como algumas crianças têm dificuldade em verbalizar seus sentimentos, o desenho lhes permite transmitir experiências (Ebersöhn et al., 2012). Vários estudos têm se utilizado dessa técnica para avaliar as interações interpessoais e relações afetivas na família (Ebersöhn et al., 2012).

Comparações clínicas realizadas entre o KFD e demais técnicas projetivas gráficas, como da Casa-ÁrvorePessoa (HTP) ou o Desenho da Figura Humana (DAP), revelaram que, enquanto no primeiro apareciam indicadores de dificuldades e conflitos nas relações familiares, nos demais se constatavam a ausência de problemas. Desse modo, infere-se que o KFD permite uma análise mais detalhada de como o sujeito é refletido e expresso na família, bem como sua percepção sobre as interações entre os membros que a compõem, de modo que a criança a represente como uma unidade ativa (Handler \& Habenicht, 1994).

Em outro estudo que teve como objetivo comparar os métodos de análise quanti e qualitativa do DAP (Draw-a-person) convencional e do KFD (Kinetic Family Drawing, versão proposta por Reynolds, 1978) revelou diferenças significativas entre grupos normal e patológico para os dois testes, sendo que o DAP e o KFD obtiveram pontuações muito similares na maioria dos indicadores de psicopatologia, revelando que o Desenho da Família Cinética pode ser considerado um teste que capta problemas emocionais, assim como o DAP (Tharinger \& Stark, 1990).

Em pesquisa comparativa entre o Desenho da Família Cinética e o Desenho da Figura Humana (HFD) mostrou que a figura humana que representa o sujeito no desenho no KFD é muitas vezes inferior quando comparada ao HFD. Isso possivelmente ocorre porque a criança, ao desenhar uma pessoa, responde ao estímulo associando-o a seu conceito de componente da imagem corporal, recorrendo a aspectos cognitivos de seu funcionamento psíquico, bem como afetivos. Já no Desenho da Família Cinética, a criança estará mais atenuada para os aspectos afetivos. Nesse contexto, pode-se inferir que a criança, ao desenhar sua família fazendo alguma coisa, concentra-se menos no que é conhecido e mais no que ela pode sentir sobre si e sobre os membros da família (Miller, 1995).
Esse instrumento possui um manual interpretativo desenvolvido por dois autores norte-americanos, Burns e Kaufman (1972), o qual contém indicadores de análise divididos em grupos (características dos desenhos e figuras, ações, estilos e símbolos). De um modo geral, o conjunto das ações presente nos desenhos se refere a um movimento de energia entre as pessoas, o qual era chamado por vários nomes, como "relações interpessoais", "amor", "libido", "sentimentos". Essa energia varia de intensidade, sendo baixa entre estranhos e em vínculos fracos, e alta entre vínculos fortes. Burns e Kaufman (1972) ressaltam que muitos dos desenhos refletem um "campo de força" dentro da imagem ou entre as figuras. Essas forças podem ser representadas em uma variedade de imagens, como bolas, barreiras ou objetos considerados perigosos. Já os estilos descritos pelos autores se referem ao modo como os indivíduos expressavam seus vínculos e sentimentos. As crianças, segundo os autores, têm uma tendência de expressarem o amor de forma natural e sem barreiras. Essa disposição seria a ideal e a não patológica. Porém outras formas de expressarem o afeto poderiam ser percebidas nos desenhos, como o compartimentalismo (linhas que separam os membros da família), encapsulamento (colocar um membro da família dentro de uma "bolha"), o aparecimento de bordas no desenho, entre outras (Burns \& Kaufman, 1972). Como última parte de análise proposta por Burns e Kaufman (1972), há o significado dos elementos que podem aparecer nos desenhos. A recorrência e a consistência do significado devem estar associadas ao histórico do paciente e demais materiais clínicos coletados (Burns \& Kaufman, 1972).

Quanto ao sistema de pontuação para a análise do $\mathrm{KFD}$, os autores não trazem, no manual interpretativo, parâmetros de cotação; e ressalta-se que não há um consenso na literatura. O que pode-se inferir é que determinados itens, ao aparecerem nos desenhos, podem estar associados a conflitos familiares e problemas emocionais; enquanto que outros itens podem estar relacionados a um vínculo familiar saudável. Desse modo, o KFD pode ser pontuado de acordo com a presença e ausência desses itens. Os itens considerados conflituosos recebem 1 ponto cada; já os itens considerados saudáveis, ao aparecerem, têm sua pontuação invertida na escala. Em um escore geral, quanto maior a pontuação total, maior a probabilidade do sujeito possuir problemas nos vínculos familiares e/ou problemas emocionais.

Salienta-se que esse manual interpretativo foi desenvolvido baseado em estudos de caso e seus indicadores não obtêm evidências de validade por meio de parâmetros psicométricos, o que torna o instrumento frágil e suscetível a críticas no meio acadêmico. Outro ponto importante a ser debatido sobre esse instrumento é o fato de que ele foi desenvolvido embasado em atendimentos clínicos de crianças e adolescentes caucasianos norte-americanos de classe média, não tendo, portanto, 
parâmetros normativos representativos para a análise de seus indicadores (Handler \& Habenicht, 1994).

No que se refere a estudos de fidedignidade para o KFD até o ano de 1994, levantados em uma revisão de literatura por Handler e Habenicht, essa técnica, de um modo geral, tem obtido graus elevados de concordância entre os juízes em seus resultados, com porcentagens médias variando entre $87 \%$ e $95 \%$. Porém, deve-se ressaltar que esses escores muitas vezes não se referem aos indicadores originais da técnica, ou seja, grande parte dos estudos se utilizou de indicadores distintos do estudo original, embasando-se em outros autores, e não no manual interpretativo. Em relação aos estudos de teste-reteste, a maior parte das variáveis do sistema de correção do KFD não mantiveram suas medidas estáveis (Handler \& Habenicht, 1994). Após esse período até o momento atual, de acordo com revisão integrativa de literatura sobre a técnica estudada realizada pelos próprios autores (Klumpp, 2017), há apenas uma pesquisa que se destacou sobre a fidedignidade do KFD. Esta foi desenvolvida por Wegmann e Lusebrink (2000), cujo objetivo principal foi descrever e validar um método de correção para o KFD com crianças de diferentes culturas. Essa pesquisa não conseguiu provar que o método de análise desenvolvido mostrasse níveis de fidedignidade estatisticamente significativos em amostras culturais diferentes.

Esse resultado enfatiza o fato de que os critérios tradicionais de pontuação acabam sendo inapropriados para alguns grupos culturais. As variações de linguagem e o que é representativo como indicador de traços latentes nos desenhos devem ser considerados de acordo com o contexto cultural de cada população investigada. Sobre esse aspecto ressalta-se o fato de o KFD ter sido submetido a estudos de tradução e adaptação transcultural de seus itens de análise para o contexto brasileiro, e que neste estudo formulou-se uma folha de correção para essa técnica autoexpressiva (Klumpp, 2017).

Já em relação ao objetivo principal da presente pesquisa, destaca-se a importância de submeter o KFD a estudos de fidedignidade, pois são fundamentais no que se refere à parâmetros psicométricos mínimos nos quais uma técnica de avaliação tem que ser submetida. A fidedignidade constitui-se como sendo um dos suportes da Psicometria que, paralelamente aos processos de normatização e de evidências de validade, asseguram a qualidade do método e a cientificidade da técnica (Villemor-Amaral, Pavan, Machado, Tavella, Cardoso, \& Miguel, 2016).

Porém, no que se refere aos testes expressivos ou projetivos, a fidedignidade torna-se mais difícil de ser evidenciada, principalmente quando comparados aos testes que possuem um conjunto de informações mais precisas e objetivas (Villemor-Amaral et al., 2016). Nesse contexto, Villemor-Amaral et al. (2016) enfatizam a necessidade de mais pesquisas que avaliem a fidedignidade dos sistemas de interpretação dos indicadores dessas técnicas, o que é um procedimento relevante e essencial para a validação desses métodos.

Urbina (2007) explica nesse contexto que corrigir ou pontuar os resultados obtidos nesse tipo de testes é uma questão a ser considerada, pois nesses casos a subjetividade do avaliador é um fator presente. A autora explica ainda que a variabilidade dos escores em um teste resultante da diferença entre avaliadores não significa negligência para o preparo das instruções para a correção do teste, e sim que essas variações têm como origem as diferenças de julgamento entre os avaliadores no que concerne à questão subjetiva envolvida nesse processo.

Sobre esta questão, Boddy (2005) explica que cada avaliador traz seu próprio estilo subjetivo para interpretar o teste autoexpressivo, o que pode afetar negativamente a confiabilidade do método. Nesse contexto explica que deve haver estudos que envolvam mais de um avaliador para pontuar um mesmo conjunto de dados coletados. Esses procedimentos podem contribuir para a minimização da questão da subjetividade que é inerente ao avaliador, permitindo controlar esse aspecto, dito como uma desvantagem do uso de instrumentos desse tipo, corroborando, desse modo, com a formação de um instrumento de avaliação autoexpressivo mais confiável e padronizado/estandardizado.

Urbina (2007) explica que esse erro de medida por conta da subjetividade pode ser controlado e estimado por um método básico que consiste na avaliação do mesmo conjunto de indicadores por dois ou mais avaliadores (fidedignidade entre avaliadores ou inter-rater agreement). As correlações encontradas nessa metodologia denominam-se "índices de fidedignidade do avaliador". Correlações significativas muito altas e positivas, como, por exemplo, no caso de índices iguais ou superiores a 0,90 , sugerem que a proporção de erro devido às diferenças entre os avaliadores é de $10 \%$ ou menos. Já estimativas baixas de fidedignidade, ou seja, as abaixo de 0,70 , sugerem que o escore derivado do instrumento investigado não pode ser considerado muito confiável, gerando probabilidades de erro de mais de 30\% nesses casos. Embora não haja um limiar mínimo consensual para que o coeficiente de fidedignidade seja considerado adequado para que o teste seja confiável, entende-se que quanto mais alto o coeficiente, melhor. A maioria dos pesquisadores e a literatura apontam valores na faixa dos 0,80 ou mais (Urbina, 2007).

Há outras maneiras de se averiguar a fidedignidade de um teste, além da fidedignidade entre avaliadores, como já mencionado. A outra forma denomina-se fidedignidade intra-avaliador, que se refere à concordância com que um mesmo juiz analisa duas vezes a mesma situação observável, após um determinado período de tempo (Naglieri, McNeish, \& Bardos, 1991). A fidedignidade intra-avaliador (intra-rater) é importante para detectar mudanças que podem ocorrer com o passar do tempo, muitas vezes inerentes ao próprio avaliador (Gregson et al., 2000). 
Outra forma refere-se à fidedignidade de teste-reteste/estabilidade temporal, que consiste em gerar estimativas sobre a quantidade de erro de amostragem relacionada ao tempo que pode afetar as pontuações do instrumento. $\mathrm{O}$ procedimento consiste na aplicação do teste na mesma amostra em duas ocasiões diferentes, separadas por um intervalo de tempo determinado pelo pesquisador de acordo com as características do instrumento avaliado (Urbina, 2007). O erro de amostragem de tempo refere-se à variabilidade inerente ao sistema de pontuação do teste, uma vez que os escores analisados pertencem a uma determinada amostra, em um determinado momento específico do tempo (Urbina, 2007).

$\mathrm{Na}$ presente pesquisa, os três tipos de fidedignidade foram averiguados, tendo como objetivo principal verificar a confiabilidade dos itens de análise do Desenho da Família Cinética.

\section{Método}

\section{Participantes}

Para a fidedignidade entre avaliadores (Etapa 1), participaram dois juízes com experiência clínica em técnicas projetivas gráficas e 50 crianças com faixa etária entre 9 a 12 anos, de ambos os sexos, de instituições de ensino públicas e privadas da região metropolitana de São Paulo, Brasil, as quais enquadravam-se nos seguintes critérios de inclusão: frequentar a escola regularmente; não possuir transtornos/patologias/síndromes registrados em documentação escolar; ter o Termo de Consentimento Livre e Esclarecido assinado pelos pais ou responsáveis e assentir com a atividade proposta. No estudo de fidedignidade intra-avaliador (Etapa 2), participou um juiz pertencente à etapa anterior. Já no estudo de estabilidade temporal (Etapa 3), participaram 16 crianças de 9 a 12 anos, todas de escola pública, moradoras da região Metropolitana de São Paulo, Brasil, as quais possuíam, além dos critérios de inclusão já apontados anteriormente na Etapa 1, problemas de aprendizagem diagnosticados em atendimento psicopedagógico de uma clínica-escola da região e nível cognitivo preservado (nível intelectual considerado normal para a idade) avaliado por teste específico.

Ressalta-se que as crianças participantes desta pesquisa diferem-se de acordo com as Etapas. As que contribuíram na Etapa 1 não precisavam possuir, necessariamente, problemas de aprendizagem, uma vez que o que estava sendo avaliado não era a precisão do instrumento em captar problemas familiares e/ou emocionais, e sim questões relacionadas à minimização da subjetividade de quem avalia, bem como o quanto o instrumento estava claro no que se referia à interpretação dos itens. Já a Etapa 3 necessitava de uma amostragem patológica, uma vez que, nesse momento, estava sendo avaliada a sensibilidade do instrumento para captar possíveis características e traços que deveriam permanecer imutáveis ao longo de um período de tempo específico ou longínquo.

Salienta-se ainda que a questão cognitiva foi uma variável que demonstrou necessidade de ser controlada nas crianças participantes da Etapa 3, uma vez que a literatura aponta que o grafismo, a composição e a elaboração dos elementos do desenho podem estar relacionados ao nível de desenvolvimento intelectual (Goodenough, 1926/1951; Koppitz, 1968; Jones, 1992). Sendo assim, problemas de ordem cognitiva poderiam afetar a execução da atividade e influenciar os resultados. Outro dado importante relacionado ao nível cognitivo é que, pelo fato do desenho captar esse aspecto, o qual não era o objetivo principal desta pesquisa, houve a necessidade de controlar essa variável, uma vez que o foco dos estudos com o KFD era o de verificar o quanto esse instrumento era sensível para capturar conflitos familiares e/ou demais problemas de caráter emocional.

\section{Instrumentos}

Para as Etapas 1 e 2 foram utilizados os seguintes instrumentos:

Desenho da Família Cinética (Kinetic Family Drawing- KFD; Burns, \& Kaufman, 1972). Esta técnica consiste no ato da criança desenhar individualmente, em uma folha branca de papel (folha tipo A4), com um lápis preto (grafite $\mathrm{n}^{\circ} .2$ ), "Uma família fazendo alguma coisa", com duração de 30 a 50 minutos.

Folha de correção do Desenho da Família Cinética (Klumpp, 2017)². É constituída por 26 itens de análise do Desenho da Família Cinética, agrupados em quatro conjuntos (1. Características esperadas nos desenhos; 2. Características das figuras; 3. Ações; 4. Estilos). $\mathrm{O}$ avaliador assinala os itens que emergem no desenho, atribuindo um (1) ponto para cada indicador presente e zero (0) para a sua ausência. Salienta-se que os itens pertencentes às características esperadas nos desenhos, que indicam um vínculo familiar saudável, devem ter seus escores invertidos. No final há a soma das pontuações, gerando um escore total. Quanto maior esse escore, maior a probabilidade de que haja conflitos familiares e/ ou problemas emocionais.

Já no estudo de estabilidade temporal (Etapa 3), além dos instrumentos já citados anteriormente, foi utilizado também o Teste das Matrizes Progressivas Coloridas de Raven para o rastreio do funcionamento cognitivo (Bandeira, Alves, Giacomel, \& Lorenzatto, 2004; Simões, 2000; Sisto, Rueda, \& Bartholomeu, 2006). Esse teste destina-se à avaliação do raciocínio geral da criança. A escala é composta por 36 itens divididos em 
3 séries: $\mathrm{A}, \mathrm{Ab}, \mathrm{B}$. Cada série possui 12 itens que estão ordenados por ordem crescente de dificuldade. A criança deve apontar ou escrever qual a figura que completa um desenho maior/padrão e/ou sequência lógica. No final os itens são pontuados gerando um escore total, o qual deve ser interpretado de acordo com uma tabela normativa para verificação do resultado.

\section{Procedimentos}

A coleta dos desenhos da Família Cinética utilizados nas Etapas 1 e 2 desta pesquisa aconteceu nas escolas das crianças participantes, em dias e horários agendados previamente, mediante autorização dos gestores e do consentimento dos pais/assentimento dos alunos. Cada aluno fez o seu desenho individualmente, de acordo com a consiga da técnica, sendo que foram agrupados em no máximo oito alunos por aplicação.

Os desenhos coletados foram enviados posteriormente aos dois juízes participantes da Etapa 1, juntamente com a Folha de correção do Desenho da Família Cinética, para que cada um realizasse, separadamente, as correções dos desenhos recebidos, de modo que fosse medida a frequência com que eles classificariam cada item, no que se refere à presença ou ausência dos indicadores de análise dos KFD coletados.

$\mathrm{Na}$ Etapa 2 um juíz pertencente à Etapa 1 efetuou a correção dos mesmos desenhos após o período de um mês. Já na Etapa 3, a aplicação do Teste das Matrizes Progressivas Coloridas de Raven e a coleta dos desenhos da Família Cinética deu-se individualmente, na Clínica-Escola, após consentimento dos pais/assentimento das crianças, utilizando-se os dias e horários das consultas de atendimento psicopedagógico dos participantes. Apenas as crianças que obtinham nível cognitivo preservado no Teste das Matrizes Progressivas Coloridas de Raven eram convidadas a fazer o Desenho da Família Cinética. As crianças que foram convidadas à desenhar o KFD fizeram o desenho novamente após o período de um mês.

Vale ressaltar que o presente estudo caracterizou-se por ser de risco mínimo a todos os participantes e que o Projeto de Pesquisa obteve aprovação no dia 01 de Outubro de 2015 pelo Comitê de Ética em Pesquisa da Instituição promotora (CNS n. 196/96), tendo seguido os preceitos éticos que regem a realização de pesquisas com seres humanos (Ministério da Saúde, Conselho Federal de Psicologia).

\section{Análise de Dados}

Os dados foram analisados por meio de estatística inferencial com o auxílio do software estatístico SPSS Statistical Package for Social Sciences (Versão 23). Nas Etapas 1 e 2. foi utilizado o Coeficiente kappa de Cohen. Já na Etapa 3 foi utilizada a correlação $\rho$ de Spearman e a diferença estatística entre as médias dos pontos por meio do Teste Wilcoxon.

\section{Resultados e Discussão}

Para uma melhor compreensão dos resultados, estes serão apresentados e discutidos de acordo com as Etapas.

\section{Etapa 1: Fidedignidade entre Avaliadores}

De acordo com os parâmetros de análise do Coeficiente kappa 100\% ( $\mathrm{n}=26)$ dos itens de análise do Desenho da Família Cinética obtiveram Índices de Concordância entre os dois avaliadores aceitáveis e significativos ( $p$-valor $<0,001)$, sendo que $50 \%(n=13)$ obteve classificação como "concordância substancial" (itens 2,3 , $5,8,9,11,13,16,22,23,24,25$ e 26), com kappa variando entre 0,63 a 0,80 ; e $50 \%(n=13)$ obteve classificação como "concordância quase perfeita a perfeita" (itens 1,4 , $6,7,10,12,14,15,17,18,19,20$ e 21), com kappa variando entre 0,82 a 1,00 .

Vale ressaltar que dentro dessa última classificação, ou seja, dos itens que apresentaram "concordância quase perfeita a perfeita", 30,76\% $(n=8)$ obtiveram "concordância perfeita", com kappa =1,000 (itens 7, 12, $14,17,18,19,20$ e 21). Isso se deve possivelmente pelo fato de que seis dos itens inseridos nessa categoria serem compostos por indicadores de análise do Desenho da Família Cinética que não estiveram presentes, ou seja, nenhum dos 50 desenhos coletados da amostra tinha esses indicadores presentes nos desenhos, o que gera probabilidade nula de erro na correção e maior concordância entre os juízes. Estes itens foram: Item 7 (membros da família desenhados na parte de trás da folha, sendo que deve ser considerada a parte da frente ou parte principal da folha aquela que apresentar o maior número de membros desenhados); item 12 (figuras em movimentos rotativos, girando); item 14 (sujeito segurando uma bola em cima da cabeça); item 17 (presença de símbolos que indiquem eletricidade no desenho, como tomadas, fios, choques); item 18 (figura passando roupas); e item 20 (presença de símbolo " $\mathrm{X}$ " no desenho).

A ausência desses indicadores nos desenhos se deve ao fato, possivelmente, de sua grande maioria representar símbolos, embora na classificação do manual interpretativo elaborado pelos autores da técnica esses itens tenham sidos categorizados em outros conjuntos, como estilos, ações e características dos desenhos. Sobre a presença desses símbolos, salienta-se que estes podem variar de acordo com a cultura de cada população, uma vez que irão aparecer objetos e figuras que tenham maior relação e significado com o contexto em que o sujeito está inserido. Por exemplo, há no Manual Interpretativo de Burns e Kaufman (1972) desenhos da Família Cinética de crianças com a figura do pai cortando grama com o aparador, o que é algo comum na população norte-americana. Porém, no Brasil, esse símbolo pode não ser um indicador de análise representativo, uma vez que não há o costume da realização dessa atividade.

Ainda sobre esse aspecto, deve-se salientar que a 
coleta de dados para este estudo foi realizada em uma amostra normal, ou seja, coletaram-se desenhos de crianças que não possuíam patologias ou demais transtornos diagnosticados. Portanto, outros estudos precisam ser realizados com amostras clínicas para que seja observada não só a possível presença destes itens, como também a frequência de ocorrência nestes grupos.

\section{Etapa 2: Fidedignidade Intra-Avaliador}

Assim como na etapa anterior referente à fidedignidade entre avaliadores, todos os itens $(n=26 ; f=100 \%)$ do Desenho da Família Cinética na análise intra-avaliador obtiveram como Índices de Concordância valores aceitáveis e significativos ( $p$-valor $<0,001)$, extraídos pelo Coeficiente kappa de Cohen.

Do total dos itens analisados, 15,39\% $(n=4)$ obteve classificação como "concordância substancial" (itens 2, 9, 11 e 16), com kappa variando entre 0,75 a 0,79 ; e $84,61 \%$ $(n=22)$ obteve classificação como "concordância quase perfeita a perfeita" (itens $1,3,4,5,6,7,8,10,12,13,14$, $16,17,18,19,20,21,22,23,24,25$ e 26), com kappa variando entre 0,82 a 1,00 .

Pode-se inferir por meio desses resultados que o instrumento de análise do Desenho da Família Cinética está dentro dos parâmetros aceitáveis no que se refere à minimização do aspecto da subjetividade de quem avalia, e que há um controle das variações normais que um examinador está suscetível quando vai corrigir um teste, principalmente por se tratar de uma técnica autoexpressiva gráfica, como, por exemplo, cometer erros causados por distrações ou pontuar o teste de modo diferenciado pelo fato de sua interpretação naquele momento do desenho lhe parecer diferente da primeira concepção que obteve em seu primeiro contato com a produção, por questões muitas vezes internas ou emocionais.

Nesse contexto é importante destacar que a avaliação psicológica se caracteriza pelos julgamentos clínicos e pela tomada de decisões do avaliador, as quais são formuladas nas incertezas, uma vez que não há como conhecer tudo acerca do indivíduo analisado, como também a avaliação sempre perpassará pelo raciocínio e subjetividade de quem avalia. Destaca-se ainda o fato de que as tarefas de avaliação exigem o uso do raciocínio para a identificação das características relevantes para a compreensão da situação. As estratégias de pensamento e formulação da tomada de decisões utilizadas podem conduzir a erros, os quais podem comprometer significativamente a qualidade da investigação e das possíveis intervenções necessárias pensadas a partir do diagnóstico feito (Simões, 1993; Suhr, 2015).

Destacam-se na Tabela 1, por meio da comparação desses resultados com os valores dos índices de concordância obtidos no procedimento de fidedignidade entre avaliadores apresentado anteriormente, os itens que obtiveram pontuações mais elevadas. É importante ressaltar que foram retirados nessa organização os itens que obtiveram pontuações elevadas por causa da ausência em todos os desenhos. Já a Tabela 2 sumariza os itens que obtiveram menores pontuações entre avaliadores e intra-avaliador.

Tabela 1

Pontuações mais Elevadas de Concordância (Fidedignidade entre Avaliadores e Intra-Avaliador)

\begin{tabular}{ccc}
\hline Item & Índice entre avaliadores & Índice intra-avaliador \\
\hline 21 & 1,00 & 1,00 \\
19 & 1,00 & 1,00 \\
1 & 0,95 & 0,96 \\
6 & 0,85 & 0,90 \\
10 & 0,87 & 0,87 \\
4 & 0,82 & 0,82 \\
\hline
\end{tabular}

Tabela 2

Menores Pontuações de Concordância (Fidedignidade entre Avaliadores e Intra-Avaliador)

\begin{tabular}{ccc}
\hline Item & Índice entre avaliadores & Índice intra-avaliador \\
\hline 9 & 0,63 & 0,79 \\
11 & 0,68 & 0,75 \\
2 & 0,70 & 0,78 \\
16 & 0,79 & 0,79 \\
\hline
\end{tabular}

Esses resultados são importantes para revelar quais são os itens considerados mais e menos vulneráveis a questões de clareza, interpretação e subjetividade de quem avalia. 


\section{Etapa 3: Fidedignidade de Teste-Reteste}

As pontuações obtidas no teste $\overline{\mathrm{X}}=6,37(D P=1,99)$ e reteste $\overline{\mathrm{X}}=5,68(D P=1,40)$ do Desenho da Família Cinética não mostraram diferenças estatisticamente significativas (valor- $z=-1,63$, valor- $p>0,05$ ). Já a correlação de Spearman entre a primeira e segunda aplicação foi de $\rho=0,62$, estatisticamente significativa (valor$-p=0,009)$, revelando que há uma relação positiva de magnitude moderada entre os resultados obtidos entre o teste e o reteste.

Esses resultados mostram que houve similaridades entre os desenhos da primeira e segunda administração, ou seja, que não houve diferenças significativas entre a primeira e a segunda vez em que a criança realizou o Desenho da Família Cinética. O procedimento de teste-reteste, assim como mencionado anteriormente, consiste em gerar estimativas sobre a quantidade de erro de amostragem relacionada ao tempo que pode afetar as pontuações do instrumento. Esse erro está relacionado às especificidades da amostra e ao construto que o teste pretende avaliar.

Os resultados mostraram que as pontuações entre as duas aplicações mantiveram-se estáveis, o que sugere um controle deste erro de amostragem de tempo para o Desenho da Família Cinética, considerando as características do grupo amostral investigado.Deve-se ter cautela em relação aos resultados encontrados, uma vez que o Desenho da Família Cinética ainda carece de pesquisas referentes aos traços latentes, de modo que o erro de amostragem de tempo necessite ainda ser verificado em cada traço que a técnica autoexpressiva é capaz de medir.

Salienta-se também que as pontuações do Desenho da Família Cinética, bem como o erro de amostragem de tempo, necessitam ser investigados em públicos-alvo de outras faixas etárias e características, analisando os resultados encontrados em diversos contextos, de modo que os resultados sejam comparados com os achados da presente pesquisa, a fim de corroborarem para que o instrumento estudado demonstre possuir propriedades psicométricas dentro de parâmetros desejáveis no que concerne à cientificidade necessária a instrumentos de avaliação.

\section{Considerações Finais}

A presente pesquisa tinha como objetivo principal realizar estudos de fidedignidade para o Desenho da Família Cinética. Apesar das dificuldades e limitações no que se refere à validação de um instrumento de caráter projetivo, essa pesquisa visava principalmente atender à solicitação, no que concerne ao rigor metodológico e cientificidade acadêmica, de submeter o Desenho da Família Cinética a critérios mínimos de validade e confiabilidade.

A fidedignidade é um dos três pilares fundamentais no que se refere às propriedades psicométricas de um instrumento de avaliação, juntamente com as evidências de validade e a normatização. Destaca-se que a fidedignidade é considerada um dos passos iniciais desse quesito, mas essencial. Foi buscando atender a esses critérios que esta pesquisa foi desenvolvida, principalmente por não haver estudos brasileiros nesse sentido com o Desenho da Família Cinética.

No que se referem aos resultados encontrados com essa pesquisa, estes apontaram para bons índices de concordância para a técnica projetiva estudada, qualificando-o como um instrumento confiável. Pode-se inferir ainda que a versão da folha de correção contendo indicadores de análise do Desenho da Família Cinética mostrou-se confiável no sentido de possuir itens estáveis e objetivos.

Ressalta-se ainda que, por se tratar de uma técnica projetiva e que a questão da subjetividade de quem avalia não pode ser totalmente controlada, e sim minimizada, os índices de concordância entre os juízes para os itens de análise do Desenho da Família Cinética mostraram-se aceitáveis, podendo-se, portanto, inferir que os objetivos desta pesquisa foram atingidos.

Em relação ao erro de amostragem de tempo investigado no procedimento de teste-reteste, destaca-se o fato do público-alvo estar frequentando na ocasião da pesquisa a Clínica de Psicopedagogia para serem diagnosticados quanto aos seus problemas de aprendizagem. Os resultados encontrados nessa etapa do estudo mostraram que houve correlação estatisticamente significativa entre as duas aplicações do instrumento, evidenciando, portanto, que não houve mudanças representativas nas pontuações dos desenhos. Seria relevante verificar se esses resultados se modificariam caso o grupo clínico estivesse no momento da intervenção. Os resultados esperados comprovariam não apenas uma correlação baixa ou sem significado estatístico, mostrando que a técnica seria capaz de captar as modificações ocasionadas pela intervenção; bem como corroborariam para a validação do tratamento empregado neste grupo clínico, confirmando a eficácia da intervenção.

Outro ponto importante que merece destaque sobre a fidedignidade de teste-reteste refere-se à escolha do grupo clínico em oposição ao normativo para a realização desse procedimento, que seguiu o modelo de um estudo internacionalmente relevante e semelhante aos processos empregados nesta pesquisa. Essa escolha não elimina a importância da aplicação desse procedimento em um grupo controle, a fim de que seja verificada a estabilidade das pontuações do Desenho da Família Cinética. Desse modo, salienta-se a necessidade da realização de teste-reteste para a técnica projetiva estudada em outras pesquisas contendo grupos controle.

De um modo geral, conclui-se que os procedimentos realizados neste estudo são apenas o primeiro passo dentre outros importantes para a validação de um instrumento de avaliação. Sendo assim, demais pesquisas utilizando os itens de análise do Desenho da Família Cinética precisam ser realizadas. 


\section{Referências}

Bandeira, D. R., Alves, I. C. B., Giacomel, A. E., \& Lorenzatto, L. (2004). Matrizes progressivas coloridas de Raven-escala especial: Normas para Porto Alegre, RS. Psicologia em estudo, 9(3), 479-486. doi: 10.1590/S1413-73722004000300016

Boddy, C. (2005). Projective techniques in market research: Valueless subjectivity or insightful reality. International Journal of Market Research, 47(3), 239-254. doi: 10.1177/147078530504700304

Burns, R. C., \& Kaufman, S. H. (1972). Action, Styles, And Symbols in Kinetic Family Drawings (KFD). An interpretative manual. New York: Brunner/Mazel.

Ebersöhn, L., Eloff, I., Finestone, M., Van Dullemen, I., Sikkema, K., \& Forsyth, B. (2012). Drawing on resilience: Piloting the utility of the Kinetic Family Drawing to measure resilience in children of HIV-positive mothers. South African Journal of Education, 32(4), $331-348$. Recuperado de http://www.scielo.org.za/pdf/saje/v32n4/02.pdf

Goodenough, F. L. (1926/1951). Test de inteligencia infantie por medio del dibujo de la figura humanal. Buenos Aires: Paidós.

Gregson, J. M., Leathley, M. J., Moore, A. P., Smith, T. L., Sharma, A. K., \& Watkins, C. L. (2000). Reliability of measurements of muscle tone and muscle power in stroke patients. Age and Ageing, 29(3), 223-228. doi: 10.1093/ageing/29.3.223

Handler, L., \& Habenicht, D. (1994). The kinetic family drawing technique: A review of the literature. Journal of Personality Assessment, 62(3), 440-464. doi: 10.1207/s15327752jpa6203_5

Jones, C. J. (1992). Human figure drawings of mild̄ly handicapped students: Learning disabled, mildly mentally retarded, emotionally disturbed, and speechlanguage impaired. Springfield: Charles C. Thomas Publishers.

Klumpp, C. F. B. (2017). Estudos de fidedignidade e evidências de validade para o Desenho da Família Cinética (Tese de doutorado). Centro Universitário FIEO-UNIFIEO, Osasco, SP, Brasil.

Koppitz, E. M. (1968). Psychological evaluation of children's human figure drawings. New York: Grune \& Stratton.

Mercado, S. N. (s.f). El Test de la Familia Kinética: Recorrido y análisis de sus aportes clínicos. Recuperado de https://s3.amazonaws.com/academia. edu.documents/35252716

Miller, L. C. (1995). Kinetic family and human figure drawings of child and adolescent sexual offenders. (Doctor of Philosophy's Thesis) School of Education at Andrews University, Michigan, Estados Unidos.

Naglieri, J. A., McNeish, T. J., \& Bardos, A. N. (1991). DAP-SPED: Draw a Person: Screening Procedure for Emotional Disturbance. Austin, Texas: Pro-Ed.

Reynolds, C. R. (1978). A quick scoring guide to the interpretation of children's Kinetic Family Drawings (KFD). Psychology in the School, 15(4), 489-492. doi: 10.1002/1520-6807(197810)15:4<489::AID-PITS2310150406>3.0.CO;2-Z

Simões, M. R. (1993). Heurísticas, enviesamentos e erros inferenciais na mecânica da avaliação psicológica. Análise Psicológica, 2 (XI), $253-$ 266. Recuperado de http://repositorio.ispa.pt/bitstream/10400.12/3013/1/1993_2_253.pdf

Suhr, J. (2015). Psychological assessment: A problem-solving approach (pp.13-31). New York: Guilford.

Simões, M. R. (2000). Investigações no âmbito da aferição do teste das Matrizes Progressivas Coloridas de Raven (MPCR). Lisboa: Fundação Calouste Gulbenkian/Fundação para a Ciência e Tecnologia. Recuperado de http://hdl.handle.net/10316/946

Sisto, F. F., Rueda, F. J. M., \& Bartholomeu, D. (2006). Estudo sobre a unidimensionalidade do teste Matrizes Progressivas Coloridas de Raven. Psicologia: reflexão e Crítica, 19(1), 66-73. doi: 10.1590/S0102-79722006000100010

Tharinger, D. J., \& Stark, K. D. (1990). A qualitative versus quantitative approach to evaluating the Draw-A-Person and Kinetic Family Drawing: A study of mood-and anxiety-disorder children. Psychological Assessment: A Journal of Consulting and Clinical Psychology, 2(4), 365. doi: 10.1037/1040-3590.2.4.365

Urbina, S. (2007). Fundamentos da testagem psicológica. Porto Alegre: Artmed.

Villemor-Amaral, A. E., Pavan, P. M. P., Machado, M. A. S., Tavella, R. R., Cardoso, L. M., \& Miguel, F. K. (2016). A Estabilidade Temporal no Teste das Pirâmides Coloridas de Pfister. Interação em Psicologia, 19(3) 365-370. doi: 10.5380/psi.v19i3.32050

Wegmann, P., \& Lusebrink, V. B. (2000). Kinetic Family Drawing scoring method for cross-cultural studies. The Arts in Psychotherapy, 27(3), 179-190. doi: 10.1016/S0197-4556(00)00051-4

recebido em maio de 2018 aprovado em setembro de 2019

\section{Sobre os autores}

Carolina Ferreira Barros Klumpp é doutora em Psicologia Educacional (Centro Universitário FIEO). Atualmente, é docente do Programa de Stricto Sensu (Mestrado) em Educação da Universidade Ibirapuera-UNIB.

Manuela Vilar é doutora em Psicologia (Universidade de Coimbra). Atualmente, é professora auxiliar da Faculdade de Psicologia e de Ciências da Educação da Universidade de Coimbra.

Marcelino Pereira é doutor em Psicologia. Atualmente, é coordenador do Mestrado Integrado em Psicologia e professor associado da Faculdade de Psicologia e de Ciências da Educação da Universidade de Coimbra.

Márcia Siqueira de Andrade é doutora em Psicologia da Educação (PUC). Atualmente, é docente do Programa de Stricto Sensu (Mestrado) em Educação da Universidade Ibirapuera-UNIB. 